\title{
LOG DEL PEZZO SURFACES OF RANK 2 AND CARTIER INDEX 3 WITH A UNIQUE SINGULARITY
}

\author{
FEI WANG
}

\begin{abstract}
Log del Pezzo surfaces play the role of the opposite of surfaces of general type. We will completely classify all the log del Pezzo surfaces of rank 2 and Cartier index 3 with a unique singularity.
\end{abstract}

The open log del Pezzo surfaces of rank one are discussed by Miyanishi and Tsunoda in [10, [11, [12]; and the (complete) log del Pezzo surfaces of rank one are studied by Kojima [6], [7], Zhang [15], [16]. Alexeev and Nikulin give the classification of the log del Pezzo surfaces of index $\leq 2$ in [1], and Nakayama gives a geometrical classification without using the theory of K3 lattices in [13].

Definition 1 ([16, Definition 1]). Let $\bar{X}$ be a normal projective surface with only quotient singularities. Then $\bar{X}$ is called a logarithmic (abbr. log) del Pezzo surface if its anti-canonical divisor $-K_{\bar{X}}$ is an ample $\mathbb{Q}$-Cartier divisor.

The smallest positive integer $I$ such that $I K_{\bar{X}}$ is a Cartier divisor is called the Cartier index of $\bar{X}$, and the Picard number $\rho(\bar{X})$ is called the rank of $\bar{X}$. For notations and terminologies, we refer to Section 1. In the present article, we will give the complete classification of the log del Pezzo surfaces of rank 2 and Cartier index 3 with a unique singularity by proving the following.

Main Theorem. Let $\bar{X}$ be a log del Pezzo surface with a unique singularity $x_{0}$, and $(X, D)$ the minimal resolution. Suppose that $\bar{X}$ has rank 2 and Cartier index 3 . Then the following assertions hold:

1) There is a contraction $\pi: \bar{X} \rightarrow \bar{Y}$ of an irreducible curve $\bar{C}$ on $\bar{X}$ to a log del Pezzo surface $\bar{Y}$ of rank 1 . Let $C$ be the proper transform of $\bar{C}$ on $X$. Then $C$ is a (-1)-curve.

2000 Mathematics Subject Classification. Primary: 14E15, Secondary: 14J26, 14 J45.

Key words and phrases. Log del Pezzo surfaces, Quotient singularities. 
2) The weighted dual graph of $C+D$ is of one of the 29 configurations in Figure 6 . Moreover, they are all realizable.

\section{Preliminaries}

We work on an algebraically closed field of characteristic zero.

Definition 2 ([5, Definition 0.2 .10$])$. Let $\bar{X}$ be a normal variety. Then $\bar{X}$ is said to have log terminal singularities if

1) the canonical divisor $K_{\bar{X}}$ is a $\mathbb{Q}$-Cartier divisor, i.e., $m K_{\bar{X}}$ is a Cartier divisor for some $m \in \mathbb{Z}^{+}$, and

2) there exists a resolution of singularities $f: X \rightarrow \bar{X}$ with irreducible exceptional divisors $\left\{D_{j}\right\}_{j=1}^{n}$ such that $D:=\sum_{j=1}^{n} D_{j}$ is a simple normal crossing divisor, and that

$$
K_{X}=f^{*}\left(K_{\bar{X}}\right)+\sum_{j=1}^{n} \alpha_{j} D_{j}
$$

for some $\alpha_{j} \in \mathbb{Q}$ with $\alpha_{j}>-1$.

Lemma 1 ([4, Theorem 9.6], [10, §4.1]). Suppose $\bar{X}$ is a normal surface. Then $\bar{X}$ has only log terminal singularities if and only if $\bar{X}$ has only quotient singularities. Moreover, if this is the case, let $X \rightarrow \bar{X}$ be the minimal resolution, then each irreducible exceptional curve is a nonsingular rational curve.

Recall that a del Pezzo surface is a normal surface with ample anti-canonical divisor. It follows from Definition 2 and Lemma 1 that, the log del Pezzo surface as in Definition 1 is equivalent to "the del Pezzo surface with only log terminal singularities".

Remark 1. Let $\bar{X}$ be a log del Pezzo surface. Since $\operatorname{dim} \bar{X}=2$, in Definition 2 we can take $f: X \rightarrow \bar{X}$ to be the minimal solution. Then $\alpha_{j} \leq 0$ for all $j$. It follows that $D^{\#}:=-\sum_{j=1}^{n} \alpha_{j} D_{j}$ is an effective $\mathbb{Q}$-Cartier divisor, and $f^{*}\left(K_{\bar{X}}\right)=K_{X}+D^{\#}$. If $\alpha_{k}=0$ for some $k$, then $\alpha_{j}=0$ for all $D_{j}$ in the connected component of $D$ containing $D_{k}$ ([9, Proposition 4-6-2]). If $D^{\#}=0$, then $f^{*}\left(K_{\bar{X}}\right)=K_{X}$ and $\bar{X}$ is a Gorenstein $\log$ del Pezzo surface, which is completely classified in [14]. The case when $\bar{X}$ has index 2 is classified in [1] and [13]. 
LOG DEL PEZZO SURFACES OF RANK 2 AND CARTIER INDEX 3 WITH A UNIQUE SINGULARITצ Lemma 2 (cf. [16, Lemma 1.1]). Let $\bar{X}$ be a log del Pezzo surface. With the notations in Remark 1, we have the following assertions:

1) $-\left(K_{X}+D^{\#}\right) \cdot C \geq 0$ for every irreducible curve $C$ on $X$, and the equality holds if and only if $C \subseteq \operatorname{Supp}(D)$.

2) If $C \nsubseteq \operatorname{Supp}(D)$ is an irreducible curve on $X$ with negative self-intersection number, then $C$ is a $(-1)$-curve.

3) $\rho(X)=n+\rho(\bar{X})$.

Proof. 1) Note that $f$ is birational. Since $-K_{\bar{X}}$ is ample,

$$
-\left(K_{X}+D^{\#}\right) \cdot C=-f^{*}\left(K_{\bar{X}}\right) \cdot C=-K_{\bar{X}} \cdot f_{*}(C) \geq 0 .
$$

The equality holds if and only if $f_{*}(C)$ is a point, i.e., $C \subseteq \operatorname{Supp}(D)$.

2) Suppose $C \nsubseteq \operatorname{Supp}(D)$. Then by (1) and the adjunction formula,

$$
0<-\left(K_{X}+D^{\#}\right) \cdot C \leq-K_{X} \cdot C=2+C^{2}-2 p_{a}(C) \leq 2+C^{2} \leq 1 .
$$

It follows that $C^{2}=-1$ and $p_{a}(C)=0$. So $C$ is a $(-1)$-curve.

3) $\mathrm{NS}_{\mathbb{Q}}(X):=\mathrm{NS}(X) \otimes_{\mathbb{Z}} \mathbb{Q}$ is generated by $f^{*}\left(\mathrm{NS}_{\mathbb{Q}}(\bar{X})\right)$ and $\left\{D_{j}\right\}_{j=1}^{n}$.

In [6], $(X, D)$ is assumed to be almost minimal, and we will show in the following that the minimal resolution of every log del Pezzo surface of rank 1 is almost minimal. Hence, we can use the classification in the paper for our discussion in Sections 24.4.

Definition $3([10, \S 3.11])$. Let $\bar{X}$ be a surface and $(X, D) \rightarrow \bar{X}$ the minimal resolution. With the notations in Remark 1, let $\operatorname{Bk}(D)=D-D^{\#}$. Then $(X, D)$ is called almost minimal if for every irreducible curve $C$ on $X$ either

1) $\left(K_{X}+D^{\#}\right) \cdot C \geq 0$; or

2) the intersection matrix of $C+\operatorname{Bk}(D)$ is not negative definite.

Lemma 3. Let $\bar{X}$ be a log del Pezzo surface of rank 1 . Then its minimal resolution $(X, D)$ is almost minimal.

Proof. Suppose there exists an irreducible curve $E$ on $X$ such that $E \cdot\left(K_{X}+D^{\#}\right)<0$ and the intersection matrix of $E+\operatorname{Bk}(D)$, i.e., of $E+D$, is negative definite.

Let $\bar{E}=f_{*}(E)$. Since $0>E \cdot f^{*}\left(K_{\bar{X}}\right)=\bar{E} \cdot K_{\bar{X}}, \bar{E}$ is a curve on $\bar{X}$. Recall that $\rho(\bar{X})=1$. We can write $\bar{E} \equiv r K_{\bar{X}}$ for some $r \in \mathbb{Q}$. Then $(\bar{E})^{2}=r^{2}\left(K_{\bar{X}}\right)^{2} \geq 0$. 
On the other hand,

$$
f^{*}(\bar{E})=E+\sum_{j=1}^{n} \beta_{j} D_{j}
$$

for some $\beta_{j} \in \mathbb{Q}$. Let $H=\sum_{j=1}^{n} \beta_{j} D_{j}$. Then

$$
(\bar{E})^{2}=\left(f^{*}(\bar{E})\right)^{2}=(E+H)^{2}<0,
$$

because the intersection matrix of $E+D$ is negative definite. This leads to a contradiction.

\section{The Types of Weighted Dual Graphs of $D$}

In this section, we assume that $\bar{X}$ is a log del Pezzo surface of Cartier index 3 with a unique singularity $x_{0}$, and use the notations in Section 11. Note that the dual graph of the exceptional divisor $D$ is of A-D-E Dynkin's type. We are going to determine all the possible types of the weighted dual graphs of $D$.

Let $a_{j}=-\alpha_{j}$. Then $f^{*}\left(K_{\bar{X}}\right)=K_{X}+\sum_{j=1}^{n} a_{j} D_{j}$ for some $0<a_{j}<1$. It is given that $3 K_{\bar{X}}$ is a Cartier divisor, so is $\sum_{j=1}^{n} a_{j} D_{j}$. Therefore, $a_{j} \in\{1 / 3,2 / 3\}$ for all $j$. Note that for each $i=1, \ldots, n$,

$$
0=f^{*}\left(K_{\bar{X}}\right) \cdot D_{i}=\left(K_{X}+\sum_{j=1}^{n} a_{j} D_{j}\right) \cdot D_{i}=-2-\left(D_{i}\right)^{2}+\sum_{j=1}^{n} a_{j}\left(D_{i} \cdot D_{j}\right)
$$

That is,

$$
\sum_{j=1}^{n} a_{j}\left(D_{i} \cdot D_{j}\right)=2+\left(D_{i}\right)^{2}, \quad i=1, \ldots, n
$$

Using these results, we can show that

Proposition 1. Let $\bar{X}$ be a log del Pezzo surface of Cartier index 3 with a unique singularity, and $(X, D)$ its minimal resolution. Then

1) the weighted dual graph of $D$ is of one of the nine cases listed in the second column of Figure 1, and

2) the possible sizes of D are given in the third column of Figure 1 .

We will leave the proof of (2) in Section 3 .

Proof of Proposition 1 (1). Consider the two cases: 
LOG DEL PEZZO SURFACES OF RANK 2 AND CARTIER INDEX 3 WITH A UNIQUE SINGULARITß

\begin{tabular}{|c|c|c|}
\hline No. & Weighted Dual graph of $D$ & Size \\
\hline I & $\begin{array}{c}\circ \\
-3 \\
-3\end{array}$ & $n=1$ \\
\hline II & $\stackrel{\circ}{-6}$ & $n=1$ \\
\hline III & 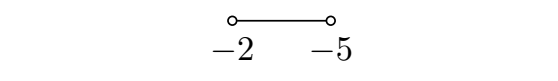 & $n=2$ \\
\hline IV & $\begin{array}{ccc}\circ & \longrightarrow & -2\end{array}$ & $n=3$ \\
\hline V & $\begin{array}{cccc}0 & -2 & -2 & -4\end{array}$ & $2 \leq n \leq 10$ \\
\hline VI & $\begin{array}{ccccc}0 & -3 & -2 & -2 & -4\end{array}$ & $3 \leq n \leq 9$ \\
\hline VII & 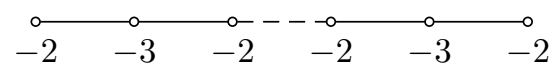 & $4 \leq n \leq 9$ \\
\hline VIII & 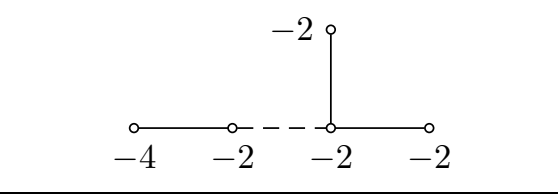 & $4 \leq n \leq 9$ \\
\hline IX & $\begin{array}{ccccc} & & & & \\
& & & & \\
-2 & -3 & -2 & -2 & -2\end{array}$ & $4 \leq n \leq 8$ \\
\hline
\end{tabular}

FiguRE 1. Weighted Dual graph of $D$

Type A. Suppose that $D$ is a linear chain $D_{1}-D_{2}-\cdots-D_{n}$.

If $n=1$, then $a_{1}\left(D_{1}\right)^{2}=2+\left(D_{1}\right)^{2}$. When $a_{1}=1 / 3,\left(D_{1}\right)^{2}=-3$, and $D$ is given by I of Figure 1, when $a_{1}=2 / 3,\left(D_{1}\right)^{2}=-6$, and $D$ is given by II.

Suppose $n \geq 2$. Then for all $i=2, \ldots, n, a_{i-1}+a_{i}\left(D_{i}\right)^{2}+a_{i+1}=2+\left(D_{i}\right)^{2}$. This implies $2-a_{i-1}-a_{i+1}=\left(D_{i}\right)^{2}\left(a_{i}-1\right) \geq-2\left(a_{i}-1\right)$, i.e.,

$$
a_{i} \geq \frac{1}{2}\left(a_{i-1}+a_{i+1}\right) \text {. }
$$

Moreover, the equality holds if and only if $\left(D_{i}\right)^{2}=-2$.

If $a_{i}=1 / 3$ for some $i=2, \ldots, n-1$, then $a_{i-1}+a_{i+1} \leq 2 / 3$ and thus $a_{i-1}=$ $a_{i+1}=1 / 3$; consequently $a_{j}=1 / 3$ for all $j=1, \ldots, n$. In particular, $1 / 3\left(D_{1}\right)^{2}+1 / 3=$ $2+\left(D_{1}\right)^{2}$. However, this would imply that $\left(D_{1}\right)^{2}=-5 / 2 \notin \mathbb{Z}$, a contradiction. So $a_{i}=2 / 3$ for some $i=2, \ldots, n-1$. If $i \leq n-2$, then $a_{i+1} \geq \frac{1}{2}\left(a_{i}+a_{i+2}\right) \geq \frac{1}{2}\left(\frac{2}{3}+\frac{1}{3}\right)=1 / 2$, 
and then $a_{i+1}=2 / 3$. It follows by induction that $a_{j}=2 / 3$ for all $j=i, \ldots, n-1$; and similarly $a_{j}=2 / 3$ for all $j=2, \ldots, i$. We consider three cases:

(i) $a_{j}=2 / 3$ for all $j=1, \ldots, n$. Then $\left(D_{1}\right)^{2}=\left(D_{n}\right)^{2}=-4$ and $\left(D_{j}\right)^{2}=-2$ for $j=2, \ldots, n-1$. This is given by $\mathrm{V}$ of Figure 1 .

(ii) $a_{1}=1 / 3$ and $a_{j}=2 / 3$ for all $j=2, \ldots, n$. For this case, if $n=2$, then $\left(D_{1}\right)^{2}=-2$ and $\left(D_{2}\right)^{2}=-5$, which is given by III; if $n \geq 3$, then $\left(D_{2}\right)^{2}=-3$, $\left(D_{n}\right)^{2}=-4$ and $\left(D_{j}\right)^{2}=-2$ for all other $j$, which is given by VI of Figure 1 .

(iii) $a_{1}=a_{n}=1 / 3$ and $a_{j}=2 / 3$ for all $j=2, \ldots, n-1$. It is impossible if $n=2$. If $n=3$, then $\left(D_{1}\right)^{2}=\left(D_{3}\right)^{2}=-2$ and $\left(D_{2}\right)^{2}=-4$, which is given by IV; if $n \geq 4$, then $\left(D_{2}\right)^{2}=\left(D_{n-1}\right)^{2}=-3$ and $\left(D_{j}\right)^{2}=-2$ for all other $j$, which is given by VII.

Type $\mathbf{D}$ and E. Suppose that $D$ is a fork. Let $D_{3}$ be the center of the fork. It intersects with three components, say $D_{1}, D_{2}$ and $D_{4}$. Then $a_{1}+a_{3}+a_{4}+a_{2}\left(D_{2}\right)^{2}=2+\left(D_{2}\right)^{2}$. There are two cases:

(i) If $\left(D_{3}\right)^{2} \leq-3$, then $1 \geq 2-a_{1}-a_{2}-a_{4}=\left(D_{3}\right)^{2}\left(a_{3}-1\right) \geq(-3)(1 / 3)=1$. We have $a_{1}=a_{2}=a_{4}=1 / 3, a_{3}=2 / 3$ and $\left(D_{3}\right)^{2}=-3$. If $D_{4}$ intersects with, say, $D_{5}$, then $2 / 3+a_{5}+1 / 3\left(D_{4}\right)^{2}=2+\left(D_{4}\right)^{2}$ implies $\left(D_{4}\right)^{2}=(3 / 2) a_{5}-2 \geq-3 / 2$, a contradiction. So $D_{4}$ is the end of a twig, and the same is true for $D_{1}$ and $D_{2}$. Therefore, for this case $n=4$ and $\left(D_{1}\right)^{2}=\left(D_{2}\right)^{2}=\left(D_{4}\right)^{2}=-2$. The weighted dual graph is by IX $(n=4)$.

(ii) If $\left(D_{3}\right)^{2}=-2$, then $a_{1}+a_{2}+a_{4}=2 a_{3}$. It follows that $a_{3}=2 / 3$ and $a_{1}+a_{2}+a_{4}=$ 4/3. After the relabeling if necessary, we have $a_{1}=a_{2}=1 / 3$ and $a_{4}=2 / 3$. Using the same argument as above, $D_{1}$ and $D_{2}$ are twigs of $D$ consisting of a single $(-2)$-curve.

We are left to determine the last twig of $D:{ }_{D_{2}}^{D_{1}}>D_{3}-D_{4}-\cdots-D_{n}$. Using the same argument as in the case of linear chain, it follows by induction that $a_{j}=2 / 3$ for all $j=4, \ldots, n-1$. There are two cases:

(ii.a) $a_{1}=a_{2}=1 / 3$ and $a_{j}=2 / 3$ for all $j=3,4, \ldots, n$. Then $\left(D_{n}\right)^{2}=-4$ and $\left(D_{j}\right)^{2}=-2$ for all $j=1, \ldots, n-1$. This is given by VIII of Figure 1 .

(ii.b) $a_{1}=a_{2}=a_{n}=1 / 3$ and $a_{j}=2 / 3$ for all $j=3,4, \ldots, n-1$. Then $n \geq 5$, $\left(D_{n-1}\right)^{2}=-3$ and $\left(D_{j}\right)^{2}=-2$ for all $j \neq n-1$. This is given by IX $(n \geq 5)$.

\section{Contraction}

From now on, we assume that $\bar{X}$ is a log del Pezzo surface of rank 2 and Cartier index 3 with a unique singularity $x_{0}$. Since $K_{\bar{X}}$ is not numerically effective, by cone theorem, 
LOG DEL PEZZO SURFACES OF RANK 2 AND CARTIER INDEX 3 WITH A UNIQUE SINGULARITY there is a $K_{\bar{X}}$-negative extremal ray $R \subseteq \overline{\mathrm{NE}}(\bar{X})$. Let $\pi: \bar{X} \rightarrow \bar{Y}$ be the contraction of $R$. Then $\bar{Y}$ is a normal projective variety of $\operatorname{dim} \bar{Y} \leq 2$ and $\pi$ has connected fibers. We will consider the three possibilities according to the dimension of $\bar{Y}$.

Case 1: $\operatorname{dim} \bar{Y}=0$. It follows that $N_{1}(\bar{X})$ is generated by some $[\bar{C}] \in R$, and thus $\rho(\bar{X})=1$. But we assumed that $\rho(\bar{X})=2$, a contradiction.

Case 2: $\operatorname{dim} \bar{Y}=1$. Then $\bar{Y}$ is a nonsingular curve. By [3, Lemma 1.3], every $\log$ del Pezzo surface is a rational surface. Then it follows from Lüroth's theorem that the base $\bar{Y}$ is rational. Therefore, $Y \cong \mathbb{P}^{1}$. We claim that

Lemma 4. With the notations above, every fiber of the contraction $\pi: \bar{X} \rightarrow \bar{Y}$ is irreducible.

Proof. Since $\bar{Y}$ is nonsingular, the contraction $\pi: \bar{X} \rightarrow \bar{Y}$ is flat, and thus every fiber has pure dimension 1 . For any point $y \in \bar{Y}$, let $\bar{F}=\pi^{-1}(y)$. Suppose $\bar{F}$ is reducible. Since $\bar{F}$ is connected, we may choose irreducible components $\bar{F}_{1}$ and $\bar{F}_{2}$ of $\bar{F}$ such that $\bar{F}_{1} \cdot \bar{F}_{2} \geq 1$. On the other hand, $\bar{F}_{1} \equiv a \bar{F}_{2} \in R$ for some $a>0$. Then by Zariski's lemma [2, Lemma 8.2], $\bar{F}_{1} \cdot \bar{F}_{2}=a\left(\bar{F}_{2}\right)^{2}<0$, a contradiction.

We continue the discussion of $\operatorname{dim} \bar{Y}=1$. Let $y_{0}=\pi\left(x_{0}\right)$ and $\bar{C}=\pi^{-1}\left(y_{0}\right)$. Then $x_{0} \in \bar{C}$, and by Zariski's lemma, $(\bar{C})^{2}=0$. Take $f:(X, D) \rightarrow \bar{X}$ to be the minimal resolution, and $C$ the proper transform of $\bar{C}$ with respect to $f$. Then $C+D=(\pi \circ$ $f)^{-1}\left(y_{0}\right)$. By Zariski's lemma again, $C^{2}<0$, and thus $C$ is a $(-1)$-curve by Lemma 2 .

Let $y \in \bar{Y} \backslash\left\{y_{0}\right\}, \bar{F}:=\pi^{-1}(y)$ and $F$ the proper transform of $\bar{F}$ with respect to $f$. Then $F=(\pi \circ f)^{-1}(y)$. So $F^{2}=0$ and $F \cdot D^{\#}=0$. We have

$$
0>\bar{F} \cdot K_{\bar{X}}=F \cdot\left(K_{X}+D^{\#}\right)=F \cdot K_{X}
$$

Then by adjunction formula, $2 p_{a}(F)-2=F \cdot\left(F+K_{X}\right)=F \cdot K_{X}<0$, and thus $p_{a}(F)=0$. By Lemma 4, $F$ is irreducible; so $F \cong \mathbb{P}^{1}$.

Let $F_{0}$ be the singular fiber of the the $\mathbb{P}^{1}$-fibration $\pi \circ f: X \rightarrow \bar{Y}$ over $y_{0}$. Then $\operatorname{Supp}\left(F_{0}\right)=C+D$. After contracting $C$ and consecutively $(-1)$-curves in $C+D, C+D$ becomes $\mathbb{P}^{1}$. In particular, note that $D$ is connected and $C+D$ is a connected simple normal crossing divisor, we have $C \cdot D=1$. Moreover,

$$
2+n=\rho(X)=10-\left(K_{X}\right)^{2}
$$


Case 3: $\operatorname{dim} \bar{Y}=2$. Then $\pi: \bar{X} \rightarrow \bar{Y}$ is birational and the exceptional curve is irreducible [8, Proposition 2.5], denoted by $\bar{C}$. Let $C$ be the proper transform of $\bar{C}$ with respect to the minimal resolution $f:(X, D) \rightarrow \bar{X}$.

Note that $\pi \circ f: X \rightarrow \bar{Y}$ contracts $C$ into a point. By negative definiteness theorem, $C^{2}<0$. So by Lemma 2, $C$ is a $(-1)$-curve. By [5, Proposition 5-1-6], $\bar{Y}$ is $\mathbb{Q}$-factorial, and it is either smooth or it has a unique log terminal singularity $y_{0}=\pi\left(x_{0}\right)$. By taking $H=-K_{\bar{X}}$ in Lemma 5 below, $-K_{\bar{Y}}$ is ample. Therefore, $\bar{Y}$ is either a smooth del Pezzo surface or a log del Pezzo surface with a unique singularity $y_{0}$. Recall that $\rho(\bar{Y})=1$. If $\bar{Y}$ is smooth, then $\bar{Y} \cong \mathbb{P}^{2}$, the projective plane.

Lemma 5. With the notations as above, for any ample divisor $H$ on $\bar{X}, \pi_{*}(H)$ is ample.

Proof. Let $\bar{H}=\pi_{*}(H)$. Then by projection formula $H=\pi^{*}(\bar{H})+a \bar{C}$ for some $a \in \mathbb{R}$. Suppose $x_{0} \in \bar{C}$. Since $f^{-1}(\bar{C})=\operatorname{Supp}(C+D)$ and that the intersection matrix of $C+D$ is negative definite, $(\bar{C})^{2}=\left(f^{*}(\bar{C})\right)^{2}<0$. If $x_{0} \notin \bar{C}$, then $(\bar{C})^{2}=C^{2}=-1$. For either case,

$$
0<H^{2}=\left(\pi^{*}(\bar{H})+a \bar{C}\right)^{2}=\left(\pi^{*}(\bar{H})\right)^{2}+a^{2}(\bar{C})^{2} \leq\left(\pi^{*}(\bar{H})\right)^{2}=(\bar{H})^{2} .
$$

Let $\bar{E}$ be an irreducible curve on $\bar{Y}$ and $\bar{E}^{\prime}$ the proper transform of $\bar{E}$ with respect to $\pi$. Then $\pi^{*}(\bar{E})=\bar{E}^{\prime}+b \bar{C}$ for some $b \in \mathbb{R}$. We can compute that

$$
0=\bar{C} \cdot \pi^{*}(\bar{E})=\bar{C} \cdot \bar{E}^{\prime}+b(\bar{C})^{2} \geq b(\bar{C})^{2}
$$

So $b \geq 0$. Then

$$
\bar{H} \cdot \bar{E}=H \cdot \pi^{*}(\bar{E})=H \cdot\left(\bar{E}^{\prime}+b \bar{C}\right)=H \cdot \bar{E}^{\prime}+b(H \cdot \bar{C}) \geq H \cdot \bar{E}^{\prime}>0 .
$$

By Nakai-Moishezon criterion, $\bar{H}$ is an ample divisor on $\bar{Y}$.

We continue the discussion of $\operatorname{dim} \bar{Y}=2$. Let $g: Y \rightarrow \bar{Y}$ be the minimal resolution. Then $\pi \circ f$ factors through $Y$; that is, there is a proper birational morphism $\mu: X \rightarrow Y$ such that $g \circ \mu=\pi \circ f$ as illustrated in Figure 2. We see that $\mu: X \rightarrow Y$ is the composite of blow-downs of $(-1)$-curves. More precisely, it is the contraction of $C$ and consecutive $(-1)$-curves in $C+D$.

Let $y_{0}=f\left(x_{0}\right)$. If $\bar{Y} \cong \mathbb{P}^{2}$, then $Y=\bar{Y}$ and $\mu(C+D)=y_{0}$. Suppose $\bar{Y}$ is a log del Pezzo surface of rank 1 with a unique singularity $y_{0}$. Then $Y$ can be further contracted 
LOG DEL PEZZO SURFACES OF RANK 2 AND CARTIER INDEX 3 WITH A UNIQUE SINGULARITY

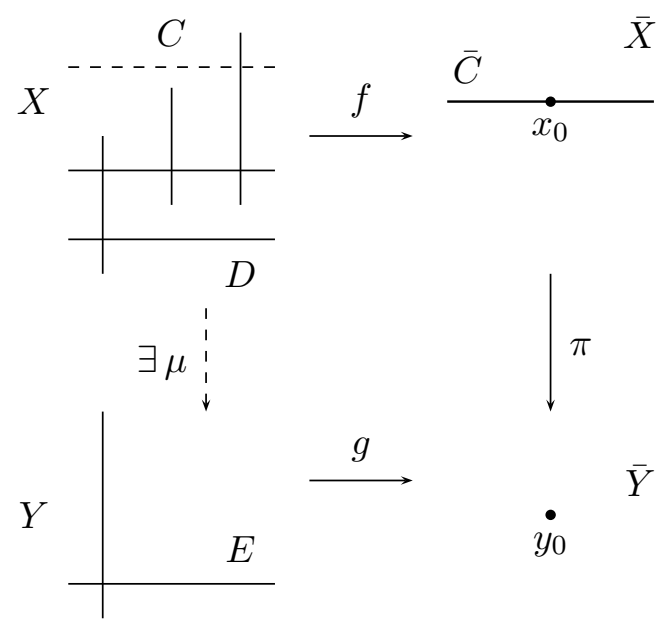

Figure 2. Divisorial Contraction

along (-1)-curves into the Hirzebruch surface $\mathbb{F}_{r}$ for some $r \geq 0[\underline{6}$, Theorem 2.1, 3.1, 4.1]. For either case,

$$
2+n=\rho(X)=10-\left(K_{X}\right)^{2}
$$

We can now determine the size of the weighted dual graphs of $D$ in Figure 1 , Proof of Proposition 1 (2). Recall that $-K_{\bar{X}}$ is ample. In particular,

$$
\begin{aligned}
0<\left(K_{\bar{X}}\right)^{2} & =\pi^{*}\left(K_{\bar{X}}\right) \cdot \pi^{*}\left(K_{\bar{X}}\right)=K_{X} \cdot \pi^{*}\left(K_{\bar{X}}\right) \\
& =K_{X} \cdot\left(K_{X}+\sum_{j=1}^{n} a_{j} D_{j}\right) \\
& =\left(K_{X}\right)^{2}+\sum_{j=1}^{n} a_{j}\left(-2-\left(D_{j}\right)^{2}\right) .
\end{aligned}
$$

For both the fiber contraction (1) and the divisorial contraction (2),

$$
2+n=\rho(X)=10-\left(K_{X}\right)^{2}<10+\sum_{j=1}^{n} a_{j}\left(-2-\left(D_{j}\right)^{2}\right) .
$$

That is, $n<8+\sum_{j=1}^{n} a_{j}\left(-2-\left(D_{j}\right)^{2}\right)$. Recall that $D^{\#}=\sum_{j=1}^{n} a_{j} D_{j}$ is evaluated explicitly in the proof of part (1), we can easily compute the possible size $n$ of $D$ :

V. $n<8+2 / 3 \cdot 2+2 / 3 \cdot 2 \Leftrightarrow n \leq 10 ;$ 


$$
\begin{aligned}
& \text { VI. } n<8+2 / 3 \cdot 1+2 / 3 \cdot 2 \Leftrightarrow n \leq 9 ; \\
& \text { VII. } n<8+2 / 3 \cdot 1+2 / 3 \cdot 1 \Leftrightarrow n \leq 9 ; \\
& \text { VIII. } n<8+2 / 3 \cdot 2 \Leftrightarrow n \leq 9 ; \\
& \text { IX. } n<8+2 / 3 \cdot 1 \Leftrightarrow n \leq 8 .
\end{aligned}
$$

This completes the proof of Proposition 1 (2).

Proof of Main Theorem. 1) Suppose $\operatorname{dim} \bar{Y}=1$. We have seen that $C+D$ can be smoothly contracted to $F \cong \mathbb{P}^{1}$ with $F^{2}=0$ along $C$ and consecutive $(-1)$-curves in $C+D$. However, by verifying all the weighted dual graphs in Figure 1, we see that none of them with any $(-1)$-curve can be contracted to such a curve, a contradiction. Therefore, $\operatorname{dim} \bar{Y}=2$ and $\bar{Y}$ is a log del Pezzo surface of rank 1. In particular, as proved in Section 3. $C$ is a $(-1)$-curve.

2) Case 1. If $\bar{Y}$ is smooth, then $Y=\bar{Y} \cong \mathbb{P}^{2}$ and $C+D$ is contracted to the smooth point $y_{0}$ along $C$ and consecutive $(-1)$-curves in $C+D$. In particular, by noting that $D$ is a simple normal crossing divisor, we have $C \cdot D=1$.

Case 2. Suppose $\bar{Y}$ is not smooth. Then $\bar{Y}$ is a log del Pezzo surface with a unique singularity $y_{0}$. Let $E$ be the exceptional divisor of the minimal resolution $g: Y \rightarrow \bar{Y}$. The configuration of $E$ is completely classified in [6, Theorem 2.1]. Recall that the possible weighted dual graphs of $D$ have been listed in Figure 1 .

(i) If $x_{0} \notin \bar{C}$, then $C$ is disjoint from $D$, and the weighted dual graphs of $D$ is the same as that of $E$.

(ii) If $x_{0} \in \bar{C}$, then $C+D$ is a connected simple normal crossing divisor since $E$ is of AD-E Dynkin's type. Note that $D$ is connected. Then $C \cdot D=1$ and $X \backslash(C \cup D) \cong Y \backslash E$. We only need to check how $C+D$ is contracted to $E$ along $C$ and consecutive $(-1)$ curves in $C+D$.

By checking all the possible weighted dual graphs of $D$ in Figure 1 and all the possible places of $C$, there are 3 configurations of $C+D(\mathrm{VI}(n=5)(\mathrm{b})$, VI $(n=6)(\mathrm{b})$, IX $(n=5)(\mathrm{b}))$ for the case when $\bar{Y}$ is smooth, and 26 configurations of $C+D$ for the case when $\bar{Y}$ is not smooth. They are given in Figure 6 .

According to the discussions above, each of these 29 possible configurations of $C+D$ can be contracted to $E$ (resp. a smooth point) along $C$ and consecutive (-1)-curves in $C+D$. There exists a $\log$ del Pezzo surface $\bar{Y}$ of rank 1 with a unique singularity (resp. 
LOG DEL PEZZO SURFACES OF RANK 2 AND CARTIER INDEX 3 WITH A UNIQUE SINGULARITIY $\bar{Y} \cong \mathbb{P}^{2}$ ), such that $E$ is the exceptional divisor of its minimal resolution $Y \rightarrow \bar{Y}$ (resp. $Y=\bar{Y}$ ). We can construct the surface $X$ by blowing up points from the corresponding surface $Y$. Let $X \rightarrow \bar{X}$ be the contraction of $D$. Then $\bar{X}$ is a projective normal surface of rank 2 and Cartier index 3 with a unique quotient singularity. We claim that

Lemma 6. For each of the configuration of $C+D$ in Figure 6 , let $\bar{X}$ be the surface defined above, then $-K_{\bar{X}}$ is ample.

It follows that $\bar{X}$ is a log del Pezzo surface of rank 2 and Cartier index 3 with a unique singularity $x_{0}$, and $D$ is the exceptional divisor of its minimal resolution $X \rightarrow \bar{X}$. In other words, every configuration in Figure 6 is realizable. We have completed the proof of Main Theorem.

\section{Ampleness of $-K_{\bar{X}}$}

In the proof of Main Theorem, for each weighted graph of $C+D$ in Figure 6, we constructed a normal projective surface $\bar{X}$ of rank 2 and Cartier index 3 with a unique quotient singularity, such that $D$ is the exceptional divisor of its minimal resolution $X \rightarrow \bar{X}$. In order to prove that $\bar{X}$ is a log del Pezzo surface, it remains to show that $-K_{\bar{X}}$ is ample (cf. Lemma 6.)

First of all, we shall evaluate $-K_{\bar{X}}$. We explore the notations used in the discussion of the divisorial contraction case in Section 3 (as illustrated in Figure 2). Recall that $\mu: X \rightarrow Y$ is the successive contraction of $(-1)$-curves in $C+D$. If $\bar{Y}$ is smooth, then $Y=\bar{Y} \cong \mathbb{P}^{2}$, and $\mu$ factors through $X \rightarrow \mathbb{F}_{1} \rightarrow Y$. If $\bar{Y}$ has a unique singularity, then $Y$ can be further contracted to the Hirzebruch surface $\mathbb{F}_{r}$ for some $r \geq 0$ along $(-1)$-curves [6, Theorem 3.1, 4.1].

We can verify the list of configurations in Figure 6 to conclude that

Lemma 7. Let $\bar{X}$ be a log del Pezzo surface of rank 2 and Cartier index 3 with a unique singularity, and $(X, D) \rightarrow X$ the minimal resolution. Then there exists a $\mathbb{P}^{1}$-fibration $X \stackrel{\Phi}{\rightarrow} \mathbb{F}_{r} \rightarrow \mathbb{P}^{1}$ with at most two singular fibers, such that one of the components $D_{\ell}$ of $D$ is a cross-section, $C$ and the other components of $D$ are contained in the singular fibers.

Then $M_{r}:=\Phi\left(D_{\ell}\right)$ is the minimal section of $\mathbb{F}_{r}$. If there are two singular fibers, let their images in $\mathbb{F}_{r}$ be $F_{1}$ and $F_{2}$. If there is only one singular fiber, let its image in $\mathbb{F}_{r}$ be 
$F_{1}$ and take $F_{2}$ to be the image of a general fiber. Take a section $N_{r} \sim M_{r}+r F_{1}$ which does not contain the image of any center of blowup. Then $-K_{\mathbb{F}_{r}}=M_{r}+N_{r}+F_{1}+F_{2}$, which form a circle (Figure [3).

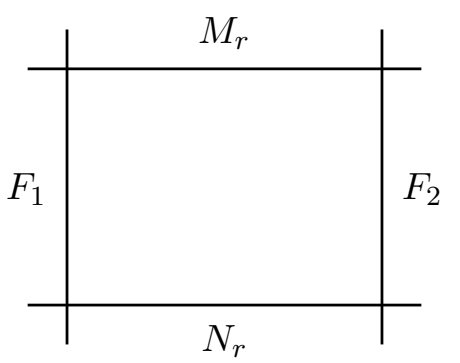

FIGURE $3 .-K_{\mathbb{F}_{r}}$

We can decompose $\Phi: X \rightarrow \mathbb{F}_{r}$ as the composite of blow-downs $X=X_{0} \stackrel{\phi_{1}}{\longrightarrow} X_{1} \rightarrow$ $\cdots \rightarrow X_{k-1} \stackrel{\phi_{k}}{\longrightarrow} X_{k}=\mathbb{F}_{r}$. Denote the exceptional curve of $\phi_{i}$ by $E_{i}, i=1, \ldots, k$. Then $K_{X_{i-1}}=\phi_{i}^{*}\left(K_{X_{i}}\right)+E_{i}$. Therefore, $-K_{X}$ can be evaluated explicitly.

Note that $-K_{X}$ is supported by $\Delta:=\Phi^{-1}\left(M_{r}+N_{r}+F_{1}+F_{2}\right)$. Let $\Delta_{+}$denote the sum of the irreducible curves which have positive coefficients appearing in $-K_{X}$. Note that $\Delta_{+}$forms a loop, and every irreducible curve in $\Delta_{+}$has coefficient 1 appearing in $-K_{X}$. In particular, the proper transforms of $M_{r}, N_{r}, F_{1}$ and $F_{2}$ on $X$ belong to $\Delta_{+}$.

Recall that in the proof of Proposition 1 (1), we computed the unique numbers $a_{j} \in\{1 / 3,2 / 3\}, i=1, \ldots, n$, such that

$$
f^{*}\left(K_{\bar{X}}\right)=K_{X}+\sum_{j=1}^{n} a_{j} D_{j}
$$

We can thus evaluate $-f^{*}\left(K_{\bar{X}}\right)$ explicitly.

The weighted dual graphs for some $-f^{*}\left(K_{\bar{X}}\right)$ are illustrated in Figures 4 and 5 . For each of the irreducible curves, the label with brackets indicates its coefficient, and the label without brackets indicates its self-intersection number. The labels for coefficient 1 are omitted. A dotted line stands for a $(-1)$-curve, and a solid line stands for a $(-2)$-curve if its self-intersection number is not indicated.

Proof of Lemma 6. From the proof of Proposition 1 (2),

$$
\left(-K_{\bar{X}}\right)^{2}=\left(K_{X}\right)^{2}+\sum_{j=1}^{n} a_{j}\left(-2-\left(D_{j}\right)^{2}\right)=8-n+\sum_{j=1}^{n} a_{j}\left(-2-\left(D_{j}\right)^{2}\right) .
$$


LOG DEL PEZZO SURFACES OF RANK 2 AND CARTIER INDEX 3 WITH A UNIQUE SINGULARITI8

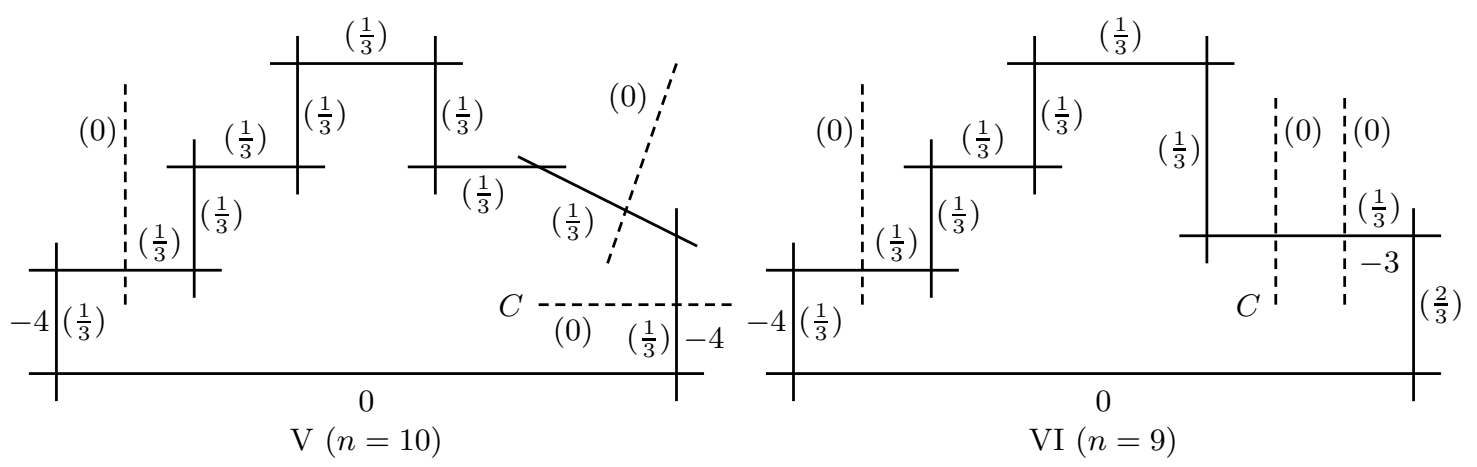

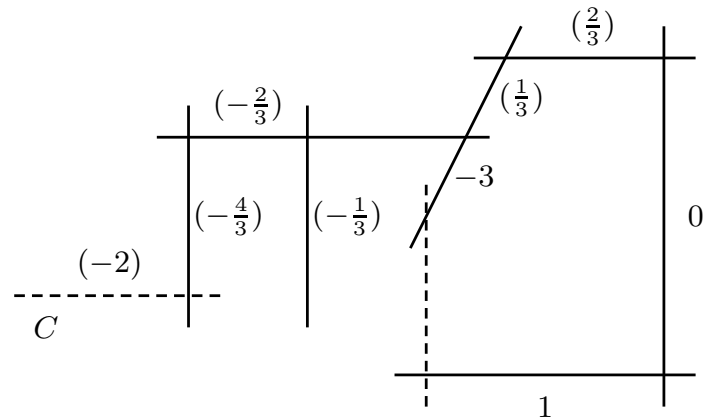

VI $(n=6)(b)$

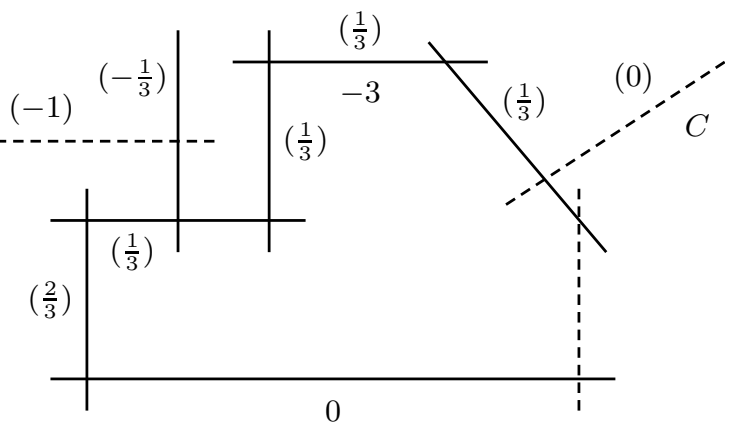

IX $(n=6)$

FiguRE $4 .-f^{*}\left(K_{\bar{X}}\right) \quad\left(c_{1}+c_{2}+r=0\right)$

The size $n$ of $D$ in Figure 1 is chosen so that $n>8+\sum_{j=1}^{n} a_{j}\left(-2-\left(D_{j}\right)^{2}\right)$. Then $\left(-K_{\bar{X}}\right)^{2}>0$. So by Nakai-Moishezon criterion, $-K_{\bar{X}}$ is ample if and only $-K_{\bar{X}} \cdot \bar{G}>0$ for every irreducible curve $\bar{G}$ on $\bar{X}$.

Let $\bar{G}$ be an irreducible curve on $\bar{X}$, and $G$ the proper transform of $\bar{G}$ on $X$. Then

$$
-K_{\bar{X}} \cdot \bar{G}=-f^{*}\left(K_{\bar{X}}\right) \cdot f^{*}(\bar{G})=-f^{*}\left(K_{\bar{X}}\right) \cdot G .
$$

We will show that this number is positive by considering the following two possibilities:

\section{$G$ is contained in a fiber.}

Case 1. Suppose $G$ is a general fiber. Then $\bar{G}$ does not contain the image of any center of blowup. So $G$ intersects with the proper transforms of $M_{r}$ and $N_{r}$ on $X$. It follows that $-f^{*}\left(K_{\bar{X}}\right) \cdot G \geq 1+1 / 3>0$.

Case 2. Suppose $G$ is contained in a singular fiber. Then $G^{2}<0$. Note that $G \nsubseteq \operatorname{Supp}(D)$. By Lemma 2, $G$ is a $(-1)$-curve. Its coefficient in $-f^{*}\left(K_{\bar{X}}\right)$ is the same as that in $-K_{X}$. 


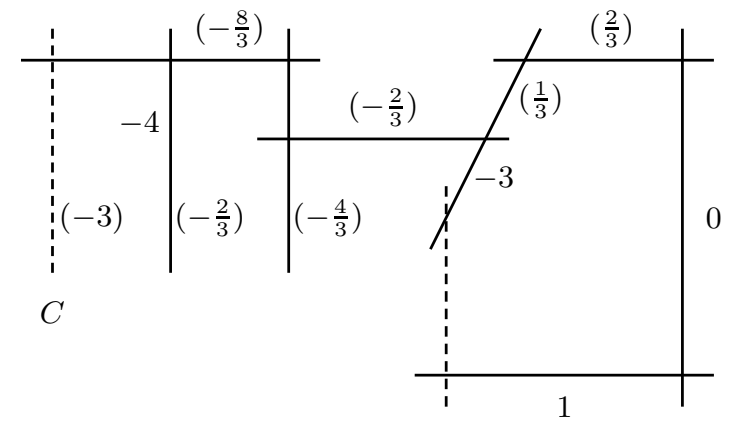

IX $(n=5)(\mathrm{b})$
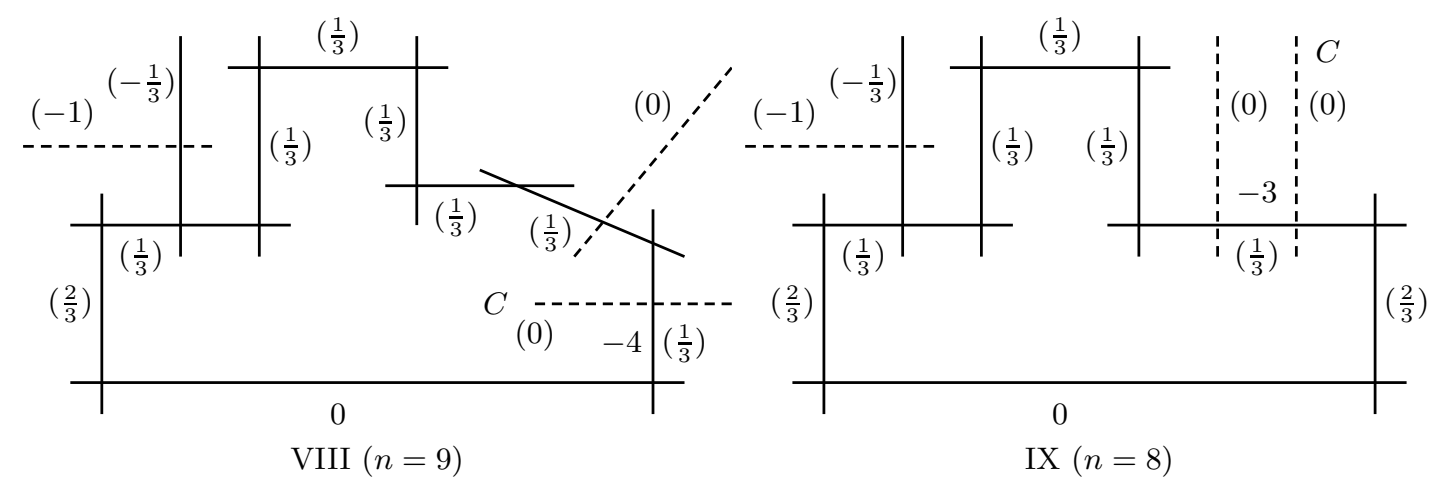

Figure 5. $-f^{*}\left(K_{\bar{X}}\right) \quad\left(c_{1}+c_{2}+r<0\right)$

(i) If $G \subseteq \operatorname{Supp}\left(\Delta_{+}\right)$, then $G$ intersects with exactly two irreducible components of $\Delta$, which are contained in $\Delta_{+}$. Moreover, exactly one of them is an irreducible component of $D$. We have $-f^{*}\left(K_{\bar{X}}\right) \cdot G \geq(-1)+1 / 3+1>0$.

(ii) If $G \nsubseteq \operatorname{Supp}\left(\Delta_{+}\right)$, let $c$ be the coefficient of $G$ in $-K_{X}$, then $G$ intersects with exactly one irreducible component of $D$, whose coefficient in $-K_{X}$ is $c+1$. Note that $G$ is disjoint from any other irreducible component of $\Delta$. So $-f^{*}\left(K_{\bar{X}}\right) \cdot G \geq$ $(-1) c+(c+1-2 / 3)>0$.

\section{$G$ is not contained in a fiber.}

Note that $G_{0}:=\Phi(G)$ is a curve in $\mathbb{F}_{r}$. Write $G_{0} \sim a M_{r}+b F_{1}$, where $a>0$ and $b \geq a r$. We have $G_{0} \cdot F_{1}=G_{0} \cdot F_{2}=a, G_{0} \cdot M_{r}=b-a r \geq 0$ and $G_{0} \cdot N_{r}=b$. Let $c_{i}$ be the smallest coefficient among all the irreducible components of $\Phi^{-1}\left(F_{i}\right)$ appearing in $-f^{*}\left(K_{\bar{X}}\right), i=1,2$. Then

$$
-f^{*}\left(K_{\bar{X}}\right) \cdot G \geq a c_{1}+a c_{2}+0+b \geq a\left(c_{1}+c_{2}+r\right) .
$$


LOG DEL PEZZO SURFACES OF RANK 2 AND CARTIER INDEX 3 WITH A UNIQUE SINGULARITIళ

By considering the sign of $c_{1}+c_{2}+r$, we have the following three cases:

Case 1. $c_{1}+c_{2}+r>0$. This is true for 22 configurations in Figure 6. For this case, it follows immediately from (3) that $-f^{*}\left(K_{\bar{X}}\right) \cdot G>0$.

Case 2. $c_{1}+c_{2}+r=0$. There are 4 configurations as given in Figure 4 ,

For this case, we may assume that $b=a r$; otherwise $b>a r$ and (3) implies that $-f^{*}\left(K_{\bar{X}}\right) \cdot G \geq a\left(c_{1}+c_{2}+r\right)+(b-a r)>0$. Then $G_{0} \sim a N_{r}$, and thus $G_{0}$ is disjoint from the minimal section $M_{r}$. Therefore, there must exist irreducible curves $L_{i} \subseteq \Phi^{-1}\left(F_{i}\right)$ with coefficient $c_{i}$ appearing in $-f^{*}\left(K_{\bar{X}}\right)$ such that $\Phi\left(L_{i}\right)$ is not a point in $M_{r}(i=1,2)$. However, it is easy to see from Figure 4 that $F_{1}$ does not exist for any of these 4 configurations.

Case 3. $c_{1}+c_{2}+r<0$. There are 3 configurations as given in Figure 5 ,

For each of them, denote $\left\{P_{i}\right\}:=M_{r} \cap F_{i}(i=1,2)$, and let $C^{\prime}, C^{\prime \prime}$ be the irreducible curves in $\Phi^{-1}\left(F_{1}\right)$ with coefficients $\leq-\left(c_{2}+r\right)$ in $-f^{*}\left(K_{\bar{X}}\right)$. Suppose that $-f^{*}\left(K_{\bar{X}}\right)$. $G \leq 0$. Then $s:=\left(C^{\prime}+C^{\prime \prime}\right) \cdot G>0$.

(i) VI $(n=6)(\mathrm{b})$. By computing the multiplicities of the center of blowups, we have $\left(F_{1} \cdot G_{0}\right)_{P_{1}} \geq 4 s$ and $\left(M_{1} \cdot G_{0}\right)_{P_{1}} \geq 4 s$. In particular, $G_{0} \sim a M_{1}+b F_{1}$ with $a \geq 4 s$ and $b \geq 8 s$. Then it would follow that $-f^{*}\left(K_{\bar{X}}\right) \cdot G \geq(-3) s+4 s+8 s>0$, a contradiction.

(ii) and (iii). VIII $(n=9)$ and IX $(n=8)$. For these cases, $\left(M_{0} \cdot G_{0}\right)_{P_{1}} \geq s$ and $\left(F_{1} \cdot G_{0}\right)_{P_{1}} \geq 2 s$. If $P_{2} \in F_{2} \cap G_{0}$, then $G_{0} \cdot N_{0} \geq\left(G_{0} \cdot M_{0}\right)_{P_{1}}+\left(G_{0} \cdot M_{0}\right)_{P_{2}} \geq s+1$. We would have $-f^{*}\left(K_{\bar{X}}\right) \cdot G \geq(-1) s+(s+1)>0$. Suppose $P_{2} \notin F_{2} \cap G_{0}$.

IX $(n=8)$ : Let $F_{2}^{\prime}$ be the proper transform of $F_{2}$ on $X$. Then $G \cdot F_{2}^{\prime}=G_{0} \cdot F_{2} \geq 2 s$. But then $-f^{*}\left(K_{\bar{X}}\right) \cdot G \geq(-1) s+(2 / 3) 2 s+s>0$, a contradiction.

VII $(n=9)$ : Note that $-f^{*}\left(K_{\bar{X}}\right) \cdot G \geq(-1) s+s=0$. If $-f^{*}\left(K_{\bar{X}}\right) \cdot G=0$, then $G_{0} \cdot M_{0}=\left(G_{0} \cdot M_{0}\right)_{P_{1}}=s$ and $G_{0} \cdot F_{1}=\left(G_{0} \cdot F_{1}\right)_{P_{1}}=2 s$; that is, $G_{0} \sim 2 s M_{0}+s F_{1}$. Note that $G$ is disjoint from $F_{2}^{\prime}$. Then $G \cdot C=2 s-G \cdot F_{2}^{\prime}=2 s$. However, this would imply that $G_{0}$ has multiplicity $2 s$ at the point $\Phi(C)$, and thus $s=G_{0} \cdot M_{0} \geq 2 s$, a contradiction again.

Therefore, $-K_{\bar{X}} \cdot \bar{G}=f^{*}\left(K_{\bar{X}}\right) \cdot G>0$ for every irreducible curve $\bar{G}$ on $\bar{X}$. Since $\left(-K_{\bar{X}}\right)^{2}>0$, by Nakai-Moishezon criterion, $-K_{\bar{X}}$ is ample for all the 29 configurations listed in Figure 6. We have completed the proof of Lemma 6. 


\section{The List of Weighted Dual Graphs of $C+D$}

I (a) and (b):

$$
\begin{array}{cc}
\circ & \circ \\
-3 & -1 \\
\circ & \circ \\
-3 & -1
\end{array}
$$

II (a) and (b):

$$
\begin{array}{cc}
\circ & \circ \\
-6 & -1 \\
\circ & \circ \\
-6 & -1
\end{array}
$$

III:

$$
\stackrel{\circ}{\circ-2} \quad-5
$$

$\mathrm{V}(n=5)(\mathrm{a})$ and $(\mathrm{b})$ :

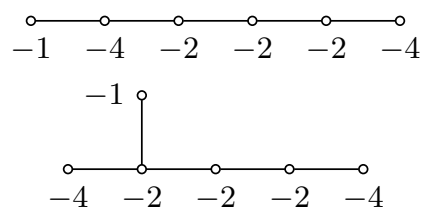

$\mathrm{V}(n=6)(\mathrm{a})$ and (b):

$$
\begin{array}{ccccccc}
0 & 0 & 0 & 0 & 0 & 0 \\
-4 & -2 & -2 & -2 & -2 & -4 & -1 \\
\circ 4 & -2 & -2 & -2 & -2 & -4 & -1
\end{array}
$$

$\mathrm{V}(n=10)$ :

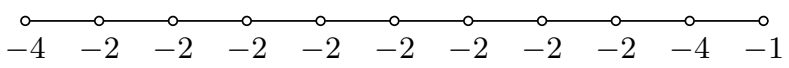

$\mathrm{VI}(n=4)$ :

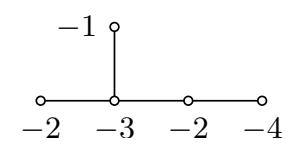

VI $(n=5)(\mathrm{a})$ and (b):

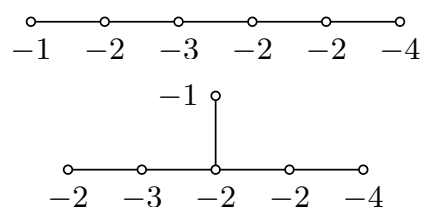

VI $(n=6)(\mathrm{a})$ and (b):

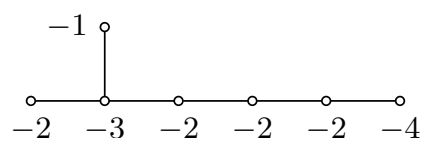


LOG DEL PEZZO SURFACES OF RANK 2 AND CARTIER INDEX 3 WITH A UNIQUE SINGULARITIY

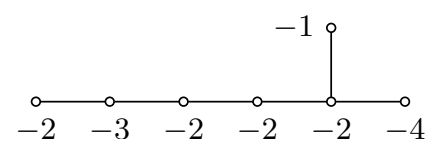

VI $(n=7)$ :

$$
\begin{array}{cccccccc}
01 & -2 & -3 & -2 & -2 & -2 & -2 & -4
\end{array}
$$

VI $(n=9)$ :

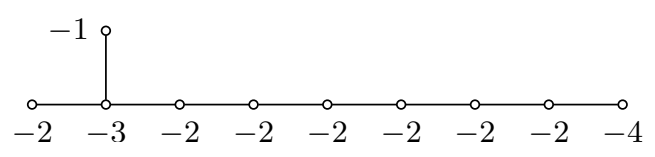

VII $(n=5)$ (a) and (b):

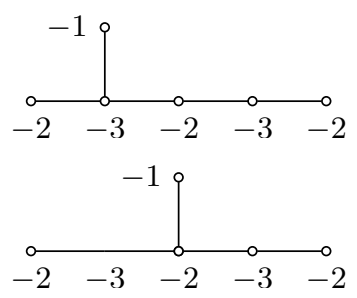

VII $(n=6)(a)$ and (b):

$$
\begin{array}{ccccccc}
\circ & 0 & 0 & 0 & 0 & 0 & \circ \\
-2 & -3 & -2 & -2 & -3 & -2 & -1 \\
\circ & 0 & \circ & 0 & \circ & \circ & 0 \\
-1 & -2 & -3 & -2 & -2 & -3 & -2
\end{array}
$$

VIII $(n=4)$ :

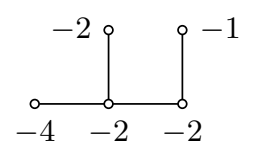

VIII $(n=5)(\mathrm{a})$ and (b):

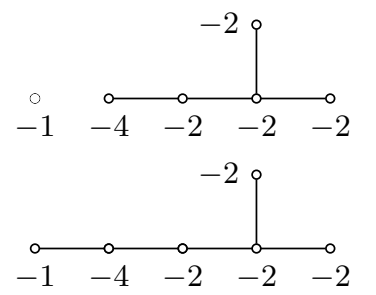

VIII $(n=9)$ :

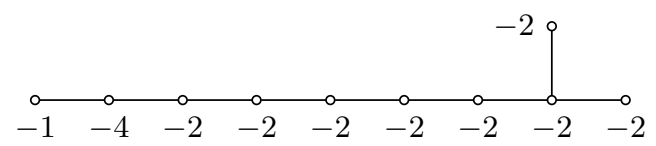

IX $(n=5)(\mathrm{a})$ and $(\mathrm{b})$ : 


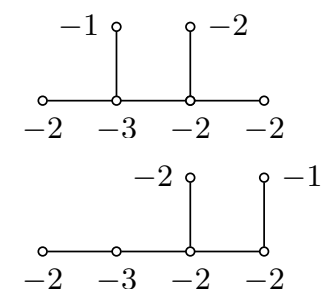

IX $(n=6)$ :

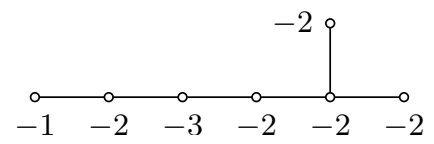

IX $(n=8)$ :

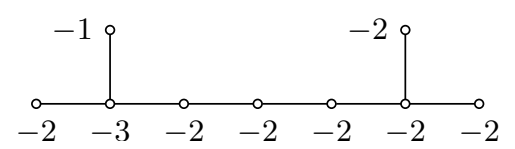

Figure 6. Weighted Dual graphs of $C+D$

Acknowledgements. The author would like to thank Prof D.-Q. Zhang for introducing the topic and his kind guidance of the paper, and thank the referee for the valuable comments.

\section{REFERENCES}

1. V.A. Alekseev and V.V. Nikulin, Classification of del Pezzo surfaces with log-terminal singularities of index $\leq 2$, involutions on $K 3$ surfaces and reflection groups in Lobachevskii spaces, Lectures in Mathematics and Its Applications 2 (1988), no. 2, 51-150.

2. W. Barth, K. Hulek, C. Peters, and A. Van de Ven, Compact complex surfaces, Ergeb. Math. Grenzgeb. (3), vol. 4, Springer-Verlag, 2004.

3. R.V. Gurjar and D.-Q. Zhang, $\pi_{1}$ of smooth points of a log del Pezzo surface is finite. I, J. Math. Sci. Univ. Tokyo 1 (1994), no. 1, 137-180.

4. Y. Kawamata, Crepant blowing-up of 3-dimensional canonical singularities and its application to degenerations of surfaces, Ann. of Math. (2) 127 (1988), no. 1, 93-163.

5. Y. Kawamata, K. Matsuda, and K. Matsuki, Introduction to the minimal model problem, Algebraic geometry, Sendai, 1985, Adv. Stud. Pure Math. 10 (1987), 283-360.

6. H. Kojima, Logarithmic del Pezzo surfaces of rank one with unique singular points, Japan. J. Math. (N.S.) 25 (1999), no. 2, 343-375.

7. _ Rank one log del Pezzo surfaces of index two, J. Math. Kyoto Univ. 43 (2003), no. 1, 101-123. 
LOG DEL PEZZO SURFACES OF RANK 2 AND CARTIER INDEX 3 WITH A UNIQUE SINGULARITIY

8. J. Kollár and S. Mori, Birational geometry of algebraic varieties, Cambridge Tracts in Math., vol. 134, Cambridge Univ. Pr., 1998.

9. K. Matsuki, Introduction to the Mori program, Universitext, Springer-Verlag, 2002.

10. M. Miyanishi, Open algebraic surfaces, CRM Monogr. Ser., vol. 12, Amer. Math. Soc., 2001.

11. M. Miyanishi and S. Tsunoda, Logarithmic del Pezzo surfaces of rank one with noncontractible boundaries, Japan. J. Math. (N.S.) 10 (1984), no. 2, 271-319.

12. _ Non-complete algebraic surfaces with logarithmic Kodaira dimension $-\infty$ and with nonconnected boundaries at infinity, Japan. J. Math. (N.S.) 10 (1984), no. 2, 195-242.

13. N. Nakayama, Classification of log del Pezzo surfaces of index two, J. Math. Sci. Univ. Tokyo 14 (2007), no. 3, 293-498.

14. Q. Ye, On Gorenstein log del Pezzo surfaces, Japan. J. Math. (N.S.) 28 (2002), no. 1, 87-136.

15. D.-Q. Zhang, Logarithmic del Pezzo surfaces of rank one with contractible boundaries, Osaka J. Math. 25 (1988), no. 2, 461-497.

16. L Logarithmic del Pezzo surfaces with rational double and triple singular points, Tohoku Math. J. (2) 41 (1989), no. 3, 399-452.

Fei Wang, Department of Mathematics, National University of Singapore, 10 Lower Kent Ridge Road, Singapore 119076

E-mail address: matwf@nus.edu.sg 